\title{
Chemiluminescence Resonance Energy Transfer Competitive Immunoassay Employing Hapten-Functionalized Quantum Dots for the Detection of Sulfamethazine
}

\author{
Mingfang Ma,${ }^{\dagger}$ Kai Wen, ${ }^{\dagger}$ Ross C. Beier, ${ }^{\S}$ Sergei A. Eremin, ${ }^{\|}$Chenglong Li, ${ }^{\dagger}$ \\ Suxia Zhang, ${ }^{\dagger,}$ Jianzhong Shen ${ }^{\dagger, *}$ and Zhanhui Wang* ${ }^{\dagger, *}$ \\ ${ }^{\dagger}$ Beijing Advanced Innovation Center for Food Nutrition and Human Health, College of \\ Veterinary Medicine, China Agricultural University, No.2 Yuanmingyuan West Road, Beijing \\ 100193, China. \\ ${ }^{\star}$ Beijing Laboratory for Food Quality and Safety and Beijing Key Laboratory of Detection \\ Technology for Animal-Derived Food Safety, No.2 Yuanmingyuan West Road, Beijing 100193, \\ China. \\ ${ }^{\S}$ Food and Feed Safety Research Unit, Southern Plains Agricultural Research Center, \\ Agricultural Research Service, United States Department of Agriculture, 2881 F\&B Road, \\ College Station, TX 77845. \\ 'Faculty of Chemistry, M. V. Lomonosov Moscow State University, Leninsky Gory, Moscow \\ 119992, Russia.
}

* Author to whom correspondence should be addressed

Tel: $+86-10-62734565$

Fax: +86-10-6273 1032

E-mail: wangzhanhui@cau.edu.cn 


\title{
Table of contents
}

\author{
1. Chemicals and Materials
}

2. Synthesis of core/multishell quantum dots

2.1. Synthesis of core quantum dots

2.2. Synthesis of $\mathrm{CdSe} / \mathrm{CdS} / \mathrm{Cd}_{0.5} \mathrm{Zn}_{0.5} \mathrm{~S} / \mathrm{ZnS}$ core/multishell quantum dots

2.3. Preparation of BS-QDs conjugates

3. Conjugation of the mAb to HRP

4. Development of the CRET-based immunoassay and ELISA in milk

5. Development of the FPIA for SMZ

5.1. Principal of FPIA for SMZ

5.2 Synthesis of the BS-AMF tracer

5.3. Optimization of tracer and antibody concentration

5.4. FPIA protocol for SMZ

5.5 Sample preparation 


\section{Chemicals and Materials}

Hexadecylanmine (HDA), 1-[3-(dimethylamino)propyl]-3-ethylcarbo-diimide methiodide (EDC) and para-iodophenol were purchased from Sigma-Aldrich (St. Louis, MO, USA). The cadmium oxide (CdO), sulfur, zinc oxide $(\mathrm{ZnO})$, selenium powder, tri- $n$-octylphosphine oxide (TOPO), oleic acid (OA), octadecylamine (ODA), 1-octadecane (ODE), and Tributyl phosphate (TBP) were obtained from TCI (Shanghai, China), 1,2-distearoyl-sn-glycero3-phosphoethanolamine- $N$-[amino(polyethylene glycol)-2000] (DSPE-PEG$\mathrm{NH}_{2}$ ) was obtained from Ruikai (Xi'an, China). Luminol, hydrogen peroxide $\left(\mathrm{H}_{2} \mathrm{O}_{2}\right)$ and horseradish peroxidase (HRP) were obtained from Beijing Keybiotech (Beijing, China). 4'-(Aminomethyl) fluorescein (4'-AMF) was obtained from Invitrogen (Carlsbad, CA, USA). All of the SAs were from Dr. Ehrenstorfer (Augsburg, Germany) and the structures of the SAs are shown in Fig. S1. The monoclonal antibody (mAb4D11), which was developed against the SAs, the control antibody (mAb5B2) developed against erythromycin (ERY), hapten BS (Figure S1) and BS-BSA were previously prepared in our laboratory. Common solvents and salts were analytical reagent grade and supplied by Beijing Reagent Corporation (Beijing, China). Pre-coated silica gel $60 \mathrm{G} \mathrm{F}_{254}$ glass plates for thin layer chromatography (TLC) were obtained from XinDe Corporation (Yantai, China). 96-well non-binding surface black and white microplates were obtained from Corning Life Sciences (Corning, NY, 
USA). Deionized water was prepared using a Milli-Q water purification system (Bedford, MA, USA).

A SpectraMax M5 microplate reader from Molecular Devices (Downingtown, PA, USA) was used to measure chemiluminescence, UV-vis absorbance, fluorescence intensity and fluorescence polarization. The morphology of the QDs was characterized using JEM-1200EX transmission electron microscopy (Jeol Ltd., Tokyo Japan). X-ray photoelectron spectra (XPS) were recorded by a Thermo ESCALAB 250XI X-ray photoelectron spectrometer (Thermo Fisher Scientific Inc., Waltham, MA, USA) with nonmonochromatized $\mathrm{Al} \mathrm{K \alpha}$ radiation as the excitation source. The tracer was confirmed by high-performance liquid chromatography-mass spectrometry. Chromatography was performed on a Waters Alliance 2690 LC system (Milford, MA, USA) and the Quattro LC triple-quadrupole mass spectrometer (Manchester, UK) was connected to the LC system via an electrospray ionization interface. 


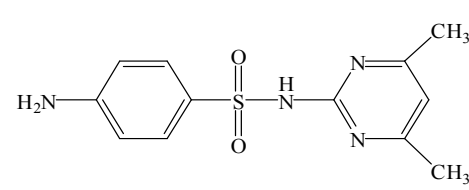

Sulfamethazine (SMZ)

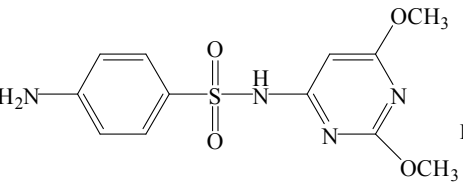

Sulfadimethoxine (SDM)

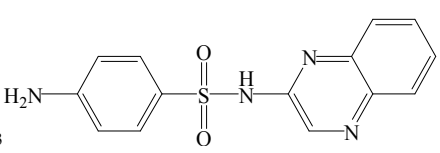

Sulfaquinoxaline (SQX)

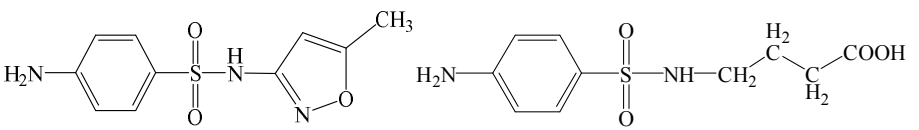

Sulfamethoxazole (SMX) 4-(4-aminophenylsulfonamido)butanoic acid (BS)

Figure S1. The chemical structure of hapten (BS) and sulfonamides used in the study. 


\section{Synthesis of core/multishell quantum dots}

\subsection{Synthesis of the CdSe core}

The highly fluorescent CdSe nanoparticles were prepared according to a previously reported method with few modifications. ${ }^{1-3}$ Briefly, the CdSe core was synthesized from $\mathrm{CdO}$ and $\mathrm{Se}$ in TOPO and HDA. For a typical reaction, a mixture of $\mathrm{CdO}(0.24$ mmol), ODE (2 g) and ODA (0.83 g) in a $25 \mathrm{~mL}$ three-necked flask was heated to 210 ${ }^{\circ} \mathrm{C}$ to obtain a colorless clear solution. TOPO $(0.6 \mathrm{~g})$ and HDA $(1.8 \mathrm{~g})$ were added to the flask under argon after the solution was cooled to room temperature. Then this mixture was reheated to $280{ }^{\circ} \mathrm{C}$ under a flow of argon. Under the same conditions, a Se precursor was prepared by dissolving Se $(2.3 \mathrm{mmol})$ in TBP $(0.472 \mathrm{~g})$ and further diluted with ODE (1.4 g) was rapidly injected into the reaction mixture, resulting in a total volume of about $25 \mathrm{~mL}$. The growth temperature was then reduced to $250{ }^{\circ} \mathrm{C}$. Generally, this step generates CdSe nanoparticles of about $3.5 \mathrm{~nm}$ in size. After the reaction was cooled down to room temperature, $2.5 \mathrm{~mL}$ hexane was slowly injected into the reaction mixture to prevent solidification of the TOPO/HDA. The extraction procedure to purify the nanocrystals from side products and unreacted precursors was

followed. ${ }^{1-3}$ The unreacted precursors were extracted into the methanol layer, and the nanocrystals remained in the hexanes/ODE layer. After centrifugation and decantation, the nanocrystals were dispersed in chloroform for further processing.

\subsection{Synthesis of the CdSe/CdS/Cd $d_{0.5} Z n_{0.5} S / Z n S$ core/multishell quantum dots}

$\mathrm{CdSe} / \mathrm{CdS} / \mathrm{Cd}_{0.5} \mathrm{Zn}_{0.5} \mathrm{~S} / \mathrm{ZnS}$ QDs were synthesized by the method of successive 
ion layer adsorption and reaction (SILAR) described previously ${ }^{4,5}$ with a slight change in reaction conditions. The CdSe nanocrystals in chloroform were added to a mixture of HDA $(0.5 \mathrm{~g})$ and TOPO $(0.5 \mathrm{~g})$ in a vessel and heated to $100{ }^{\circ} \mathrm{C}$ for $40 \mathrm{~min}$ under a vacuum. The system was held at $100{ }^{\circ} \mathrm{C}$ under vacuum for 30 min to remove the chloroform and other undesired materials with low vapor pressure and then it was cooled to room temperature. Subsequently, the solution was heated to $235{ }^{\circ} \mathrm{C}$ under a flow of argon. This was followed by the alternating addition of $\mathrm{Cd}$ and $\mathrm{Zn}$ precursors (in that order) to obtain the multishell QDs with a similar diameter as the CdSe-core particles and high photoluminescence quantum yield (QY). We covered the CdSe core particles $\left(1 \times 10^{-4} \mathrm{mmoL}\right)$ with 3 layers of $\mathrm{CdS}, 1$ layer of $\mathrm{Cd}_{0.5} \mathrm{Zn}_{0.5} \mathrm{~S}$ and 2 layers of $\mathrm{ZnS}$. And the volume of precursors was the following: $1.1 \mathrm{~mL}, 1.2 \mathrm{~mL}$ and $2.1 \mathrm{~mL}$ for the $1^{\text {st }}, 2^{\text {nd }}$ and $3^{\text {rd }}$ layers, respectively; and $1.7 \mathrm{~mL}$ for the $4^{\text {th }}$, and $2.4 \mathrm{~mL}$ and $2.9 \mathrm{~mL}$ for the $5^{\text {th }}$ and $6^{\text {th }}$ layers. The amounts of injected precursors were calculated based on the calculation of the number of surface atoms. ${ }^{6}$ After completion of the synthesis, the reaction was cooled to $70{ }^{\circ} \mathrm{C}$ and chloroform was added to prevent solidification of TOPO/HDA. The clear solution was transferred to centrifugation vials, and acetone was added until an opalescence appeared. Then the mixture was centrifuged at $2700 \mathrm{~g}$ for about $10 \mathrm{~min}$. The supernatant was discarded and the precipitate was dissolved in toluene, and stored at $4{ }^{\circ} \mathrm{C}$ under argon.

Hydrophilic QDs were prepared with amphiphilic polymers, DSPE-PEG-NH $\mathrm{NH}_{2}$ by the reverse-phase evaporation method. ${ }^{5}$ In a typical procedure, core/multishell QDs and polymers were mixed in chloroform and stirred overnight at room temperature 
(the molar ratio of QDs:polymer was about 1:40). They were dissolved in an aqueous $\mathrm{KOH}$ solution ( $\mathrm{pH} \mathrm{12)} \mathrm{and} \mathrm{then} \mathrm{the} \mathrm{QD-polymer} \mathrm{solution} \mathrm{was} \mathrm{slowly} \mathrm{evaporated.} \mathrm{To}$ remove the polymer, an excess of chloroform was added dropwise to the basic aqueous QD solution, and a white precipitate of amphiphilic polymer formed at the chloroform-water interface. Then the mixture was centrifuged at $2500 \mathrm{~g}$ for $15 \mathrm{~min}$ at room temperature, and the aqueous phase was collected. The addition of chloroform was repeated 4 times until a white precipitate was not observed. The residue of chloroform was removed under reduced pressure. Finally, the residue was transferred to water and purified by ultracentrifugation at $200,000 \mathrm{~g}$, and the DSPE-PEG-NH modified core/multishell QDs were obtained.

\subsection{Preparation of $B S-Q D$ conjugates}

To conjugate QDs with BS, $1 \mathrm{~mL}$ of $1.0 \mu \mathrm{M}$ DSPE-PEG-NH${ }_{2}$ modified core/multishell QDs and $100 \mu \mathrm{L}$ of $\mathrm{EDC} \cdot \mathrm{HCl}\left(16 \mathrm{mg} \mathrm{mL} \mathrm{m}^{-1}\right)$ were mixed with $0.46 \mathrm{mg}$ BS (dissolved in $100 \mu \mathrm{L}$ DMSO) in $1 \mathrm{~mL}$ of PBS (10 mM, pH 7.4). Then the coupling reaction was performed with gentle agitation for $2 \mathrm{~h}$ at room temperature. The BS-QDs conjugate was purified using a $10 \mathrm{~K}$ intercept molecular weight ultrafiltration tube. 


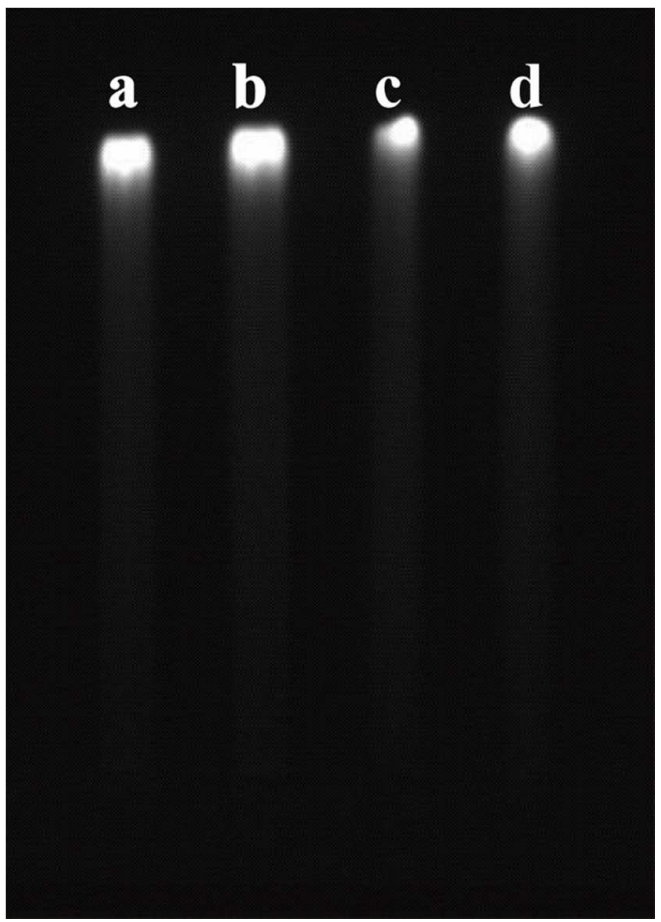

Figure S2. Photographic images of gel electrophoresis of BS-QD conjugates and QDs; a, b and c, d represent BS-QDs and QDs, respectively.

\section{Conjugation of the mAb4D11 to HRP}

The anti-SA mAb4D11 was pre-purified using saturated ammonia sulfate, and then further purified using a Sephadex G25 column. The purified mAb was conjugated to HRP by the glutaraldehyde method according to our previous report. $^{7}$ Briefly, $5 \mathrm{mg}$ of HRP was dissolved in $1 \mathrm{~mL}$ of distilled water. A newly prepared solution of $0.2 \mathrm{~mL}$ of $0.1 \mathrm{M} \mathrm{NaIO}_{4}$ was added to the HRP solution and the mixture was stirred in the dark for $20 \mathrm{~min}$ at room temperature. Then, the reaction mixture was loaded into a dialysis bag and dialyzed in sodium acetate buffer $(1 \mathrm{mM}$, $\mathrm{pH} 4.4)$ at $4{ }^{\circ} \mathrm{C}$ overnight. The mAb4D11 (10 mg) was dissolved in $1 \mathrm{~mL}$ of carbonate buffer $(10 \mathrm{mM})$ and the $\mathrm{pH}$ value of this solution was adjusted to $9.0-9.5$ by adding $20 \mu \mathrm{L}$ of carbonate buffer ( $0.2 \mathrm{M}, \mathrm{pH} 9.5)$. This basic solution was then immediately 
added to the reaction mixture and stirred in the dark for 2 hours at room temperature. Newly prepared $\mathrm{NaBH}_{4}\left(0.1 \mathrm{~mL}, 4 \mathrm{mg} \mathrm{mL}{ }^{-1}\right)$ was added, and the reaction was stirred at $4{ }^{\circ} \mathrm{C}$ for 2 hours and dialyzed in PBS (10 mM, pH 7.4) at $4{ }^{\circ} \mathrm{C}$ overnight. An equal volume of saturated ammonium sulfate solution was added dropwise with stirring at $4{ }^{\circ} \mathrm{C}$ for 1 hour followed by centrifugation at $2000 \mathrm{~g}$ for $15 \mathrm{~min}$, and the supernatant was discarded. The precipitate was washed twice with half-saturated ammonium sulfate, and a small amount of PBS was added to the precipitate. Finally, the above mixture was dialyzed in PBS at $4{ }^{\circ} \mathrm{C}$ overnight, and centrifuged at $5000 \mathrm{~g}$ for $30 \mathrm{~min}$ to remove the residual precipitate, the supernatant was collected and placed at $-20^{\circ} \mathrm{C}$ until used.

Results: The SDS-PAGE of the purified anti-SAs mAb and mAb-HRP is shown in Figure S3A and the vast majority of labelled product in lane c was HRP labelled antibody. The immunochemical activity of the mAb-HRP was then confirmed by ELISA, indicating that the mAb-HRP could specifically bind BS-OVA and SMZ (Figure S3B).

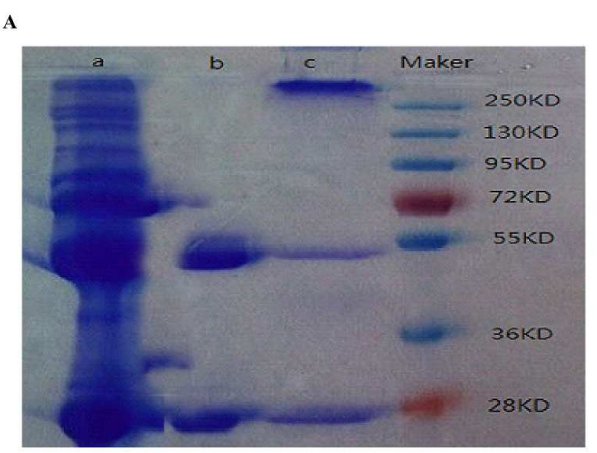

B

Figure S3. (A) SDS-PAGE, (a) Purified anti-SAs mAb by ammonia sulfate precipitation, (b) Purified anti-SAs mAb by a HiTrap Protein G column, and (c) The HRP labelled anti-SAs mAb; and (B) Immunochemical activity of mAb-HRP was confirmed by ELISA. 


\section{Development of the CRET-based immunoassay and ELISA in milk}

The CRET-based immunoassay was conducted by the following procedure. Typically, SMZ $(50 \mu \mathrm{L})$ at different concentrations, $0,0.01,0.1,1,5,10,50$, 100, 300, $500 \mathrm{ng} \mathrm{mL}^{-1}$ and mAb-HRP $(75 \mu \mathrm{L})$ were added to the wells and incubated for $10 \mathrm{~min}$ at room temperature. Then $50 \mu \mathrm{L}$ of the working concentration of the BS-QDs were added to each well and incubated for another $15 \mathrm{~min}$. Herein, the luminol/ $\mathrm{H}_{2} \mathrm{O}_{2}$ reaction catalyzed by mAb-HRP was conducted, and $p$-iodophenol acted as an enhancer. The reaction buffer $(75 \mu \mathrm{L})$ was added to each well and the chemiluminescence signals were measured at $610 \mathrm{~nm}$ after $2 \mathrm{~min}$. The ELISA procedure for SMZ was described in our previous report. ${ }^{8}$

Milk samples were provided by the National Veterinary Drug Safety and Evaluation Center (Beijing, China), which confirmed that no SAs were used during lactation and the samples were stored in the refrigerator $\left(4^{\circ} \mathrm{C}\right)$ until used. The milk samples were defatted with trichloroacetic acid (TCA). Briefly, $4 \mathrm{~mL}$ of milk was transferred to a 10 $\mathrm{mL}$ centrifuge tube containing $4 \mathrm{~mL}$ of $1.5 \% \mathrm{TCA}$. The samples were fully mixed by agitation on a shaker for $2 \mathrm{~min}$ at room temperature and then centrifuged at $1000 \mathrm{~g}$ for 10 min at $4{ }^{\circ} \mathrm{C}$ to remove the fat and protein in the upper layer. The supernatants were finally diluted with assay buffer and the samples were ready for measurement. 

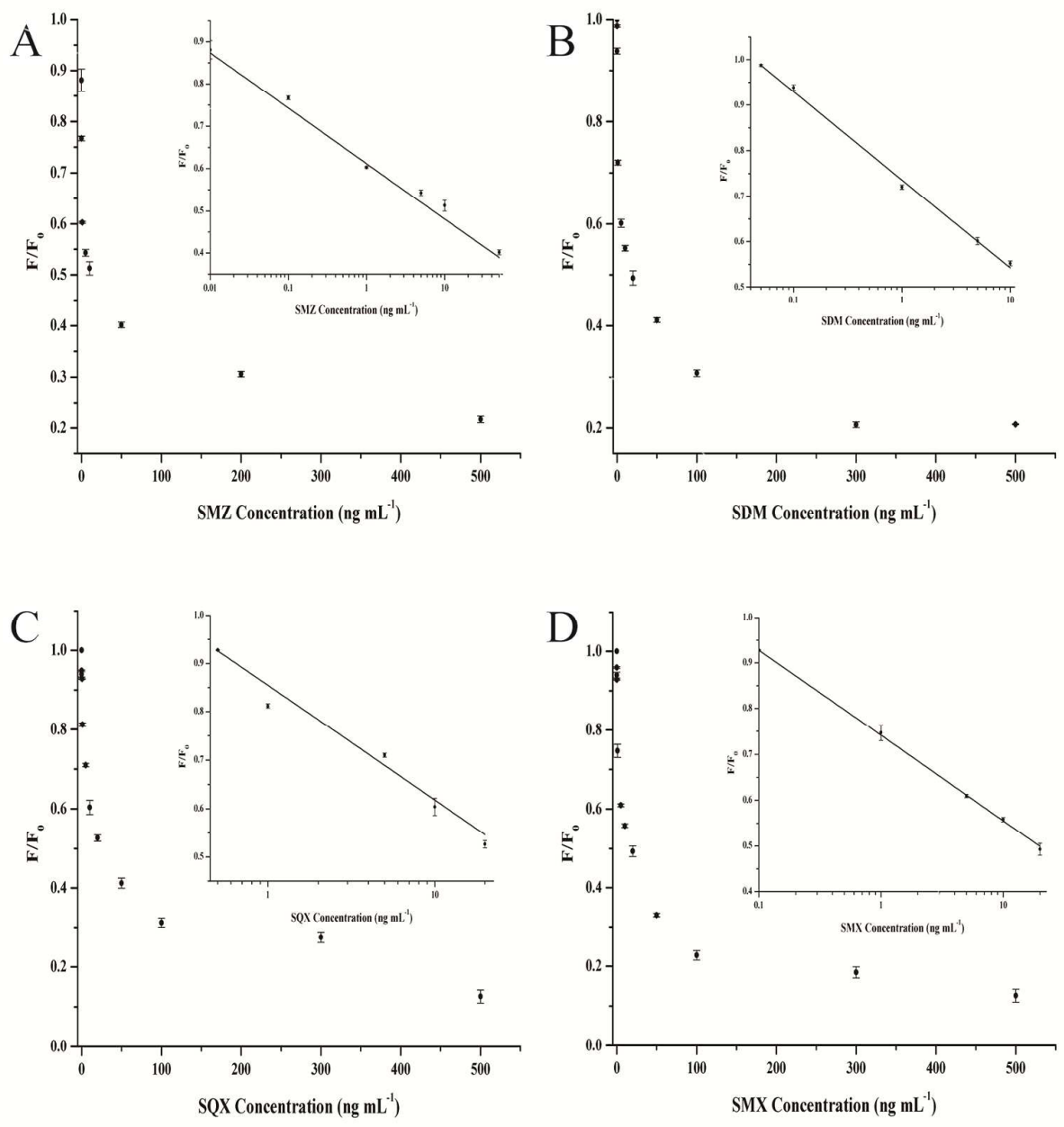

Figure S4. The standard curves of the CRET-based immunoassay for (A) SMZ, (B) SDM, (C) SQX and (D) SMX.

\section{Development of the FPIA for SMZ}

\subsection{Principal of FPIA for SMZ}

FPIA is a competitive homogeneous assay in solution phase based on differences in fluorescence polarization (FP) of the fluorescent-labeled analyte (tracer) in the antibody-bound and non-bound fractions. In a fixed reacting environment, the FP value is decided by the size of fluorescent-labeled tracer. For an unbound tracer with a small size and fast Brownian rotation, the FP value is low; however, for the 
tracer-antibody complex, the FP value is high. As shown in Fig. S5, if the concentration of analyte is high, abundant antibody will be occupied by the analyte and a certain number of tracer molecules will be free. Thus, the FP of the reaction system will be low. On the contrary, the more the tracer will combine with the antibody the higher the FP value. In a homogeneous immunoassay, an immunoreaction will reach equilibrium in minutes or even seconds, and no separation or washing steps are required. Thus, the FPIA method is a simpler and faster analytical method, which is suitable for screening large numbers of samples. ${ }^{9}$

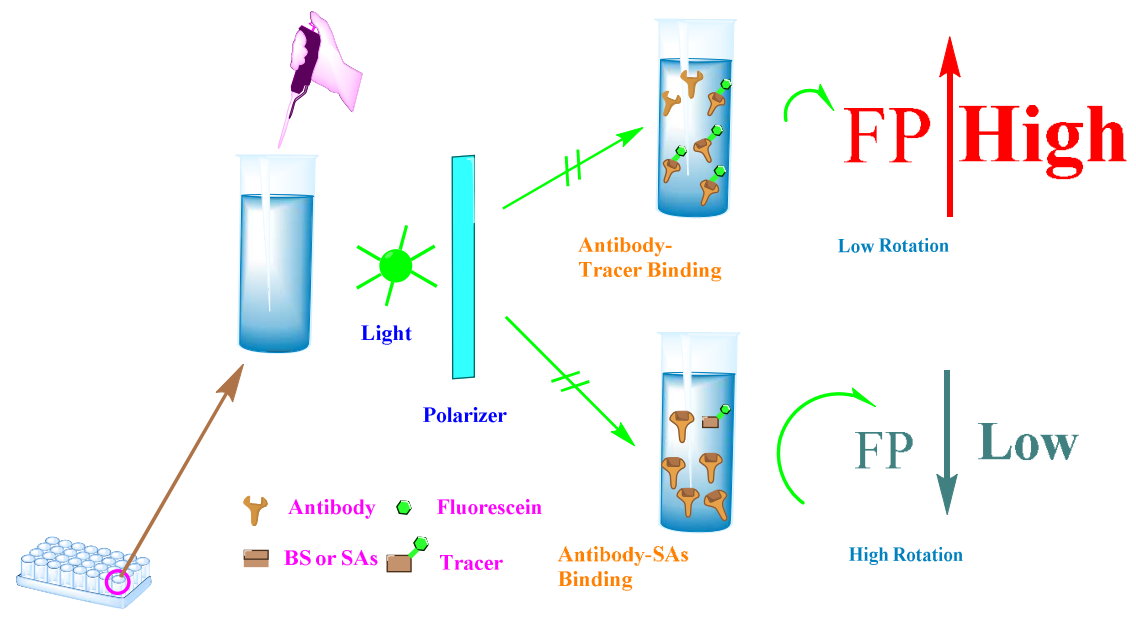

Figure S5. Detection of SMZ by fluorescence polarization immunoassay (modified from reference $^{10}$ )

\subsection{Synthesis of the BS-AMF tracer}

The carboxylic group of BS (5 mg) was activated by gently stirring for $24 \mathrm{~h}$ at room temperature in $0.5 \mathrm{~mL}$ DMF containing $3 \mathrm{mg}$ of NHS and $5 \mathrm{mg}$ of DCC. Then the mixture was centrifuged (3000 $\mathrm{g}$ for $10 \mathrm{~min}$ ) to remove the precipitated dicyclohexylurea. The clear supernatant phase was collected and $250 \mu \mathrm{L}$ of the 
supernatant was added to $4.8 \mathrm{mg}$ of 4'-AMF in $0.5 \mathrm{~mL}$ DMF. After stirring for 12 hours, a small portion of the reaction mixture was purified by TLC with methanol/AcOEt/ $\mathrm{NH}_{3} \cdot \mathrm{H}_{2} \mathrm{O}(2 / 1 / 0.04, \mathrm{v} / \mathrm{v} / \mathrm{v})$ as the eluent. The main yellow band at $\mathrm{R}_{\mathrm{f}}$ 0.6 was scraped from the plate and extracted with $0.5 \mathrm{~mL}$ of methanol to give the fluorescent conjugate BS-AMF.

\subsection{Optimization of tracer and antibody concentration ${ }^{11}$}

The tracer solution was diluted with borate buffer to concentrations ranging from approximately 0.01 to $0.4 \mathrm{nM}$. Four replicates at each concentration of tracer were performed and measured by fluorescence and fluorescence polarization. Anti-SAs mAb4D11 was serially two-fold diluted over the range of $1 / 10$ to $1 / 100000$ in borate buffer. Then $50 \mu \mathrm{L}$ of diluted antibody solution was added to each micro-plate well containing $50 \mu \mathrm{L}$ of the tracer working solution and $50 \mu \mathrm{L}$ of borate buffer for a total of $150 \mu \mathrm{L}$ per well. The mixtures were incubated for $5 \mathrm{~min}$, and then the FP readings were made.

\subsection{FPIA Protocol for SMZ}

The tracer $(50 \mu \mathrm{L})$ was mixed with $\operatorname{SMZ}(50 \mu \mathrm{L})$. Subsequently, $50 \mu \mathrm{L}$ of the optimal antibody dilutions were added, and the mixtures were shaken for 10 seconds. After an appropriate incubation time at room temperature, FP was measured at $\lambda_{\mathrm{ex}}=$ $485 \mathrm{~nm}, \lambda_{\mathrm{em}}=530 \mathrm{~nm}$ (emission cutoff $=515 \mathrm{~nm}, \mathrm{G}$ factor $\left.=1.0\right)$ and plotted against the concentration of analyte to obtain the FPIA calibration curve. The four-parameter 
logistic equation as defined below was used to fit the sigmoidal curve using OriginPro 7.0 software:

$$
\mathrm{Y}=(\mathrm{A}-\mathrm{D}) /\left[1+(\mathrm{x} / \mathrm{C})^{\mathrm{B}}\right]+\mathrm{D}
$$

Where $\mathrm{A}$ and $\mathrm{D}$ are the maximal and minimal $\mathrm{mP}$, respectively, $\mathrm{B}$ corresponds to the slope of the sigmoidal curve, and $\mathrm{C}$ is the SMZ concentration at $50 \%$ tracer binding $\left(\mathrm{IC}_{50}\right)$. The limit of detection (LOD) was defined as the mean concentration of 20 blank controls associated with 3 SD. Cross-reactivity (CR) was calculated according to the following equation:

$$
\mathrm{CR}(\%)=\left(\mathrm{IC}_{50} \text { of } \mathrm{SMZ} / \mathrm{IC}_{50} \text { of compound }\right) \times 100
$$

Where the $\mathrm{IC}_{50}$ values were obtained from calibration curves plotted for $\mathrm{SMZ}$ and other sulfonamides.

\subsection{Sample preparation ${ }^{11}$}

Four milliliter of milk were fortified with the appropriate SMZ standard solution at 100,150 , and $200 \mu \mathrm{g} \mathrm{L}^{-1}$. An equal volume of saturated ammonium sulfate solutions was added followed by mixing in a vortex mixer and sonication for $5 \mathrm{~min}$. The mixtures were then deproteinized through centrifugation for $10 \mathrm{~min}\left(10000 \mathrm{~g}\right.$ at $\left.4{ }^{\circ} \mathrm{C}\right)$. The clear supernatant $(500 \mu \mathrm{L})$ was filtered through a $0.45 \mu \mathrm{m}$ filter and $50 \mu \mathrm{L}$ of the filtrate was determined by the FPIA.

\section{Results:}

To develop the FPIA for SMZ, the tracer BS-AMF (Figure S6A) was synthesized and characterized by mass spectrometry and evaluated in the FPIA. It can be seen in 
Figure S6B that the molecular ion peak $(\mathrm{m} / \mathrm{z})$ of BS-AMF was 602.15 in the positive ion mode, indicating that AMF was successfully conjugated with BS. The results in Figure S6C demonstrated that the obtained BS-AMF had good immunochemical activity and could be used to develop a FPIA for the detection of SMZ.

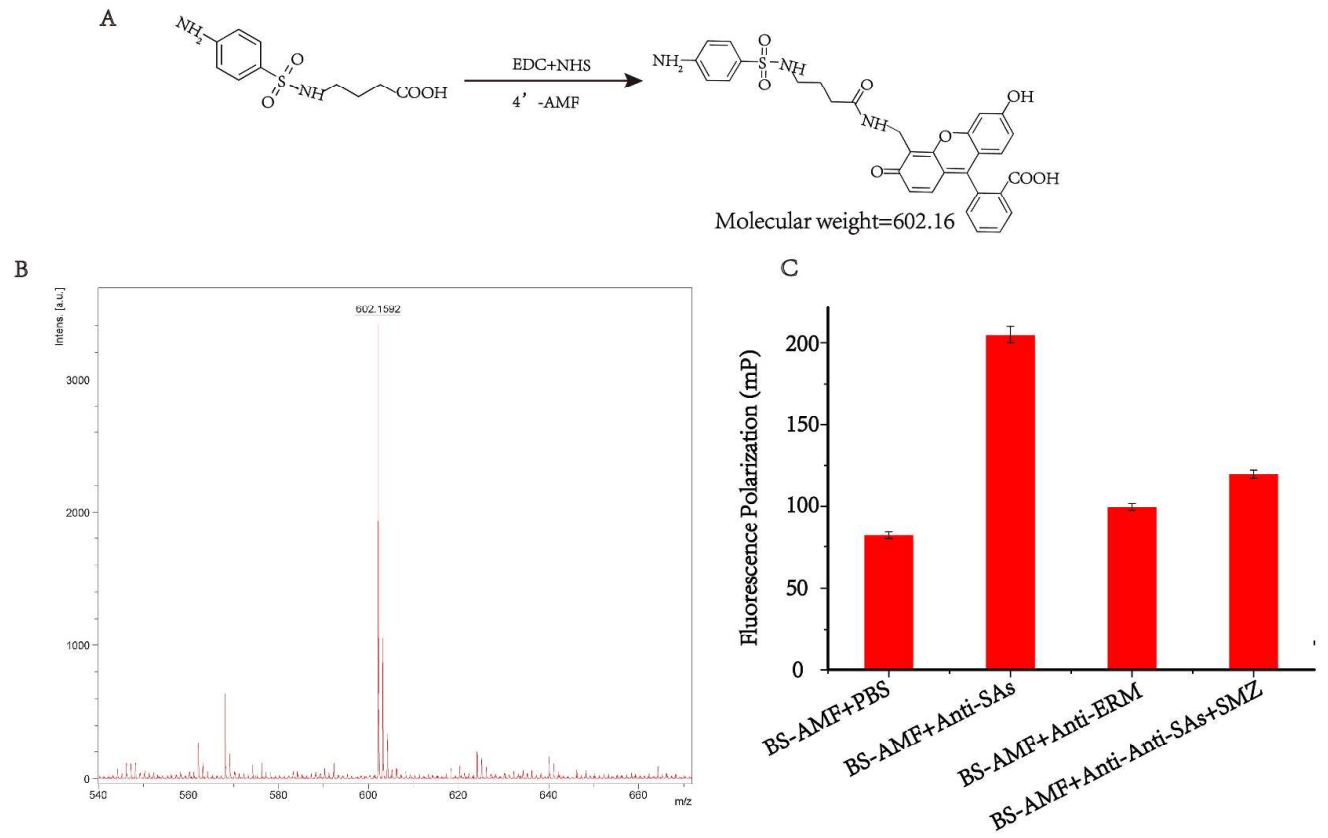

Figure S6. (A) The synthesis of tracer BS-AMF, (B) characterization of the tracer by MS, and (C) immunochemical activity of BS-AMF characterized by FPIA.

According to our previous report, $0.1 \mathrm{nM}$ of the tracer concentration giving $\sim 50$ RFU fluorescence intensity, approximately 20 times over the background signal (2 3 RFU) for borate buffer, was used in all experiments.

BS-AMF and Anti-SAs mAb4D11 were used to make the antibody dilution curve (Figure S7A) to determinate antibody titer, which is the dilution giving $70 \%$ binding of tracer, corresponding to $1 / 500$ dilution of the antibody $\left(150 \mathrm{ng} \mathrm{mL}^{-1}\right)$. The standard curve established by using $0.1 \mathrm{nM}$ BS-AMF tracer with $1 / 500$ antibody dilution (giving $70 \%$ of binding for tracer) is shown in Figure $\mathrm{S} 7 \mathrm{~B}$, where the $\mathrm{IC}_{50}$ was 
observed at $98.7 \mathrm{ng} \mathrm{mL}^{-1}$, the LOD at $12.1 \mathrm{ng} \mathrm{mL}^{-1}$ and working range of $32-145 \mathrm{ng}$ $\mathrm{mL}^{-1}$

A

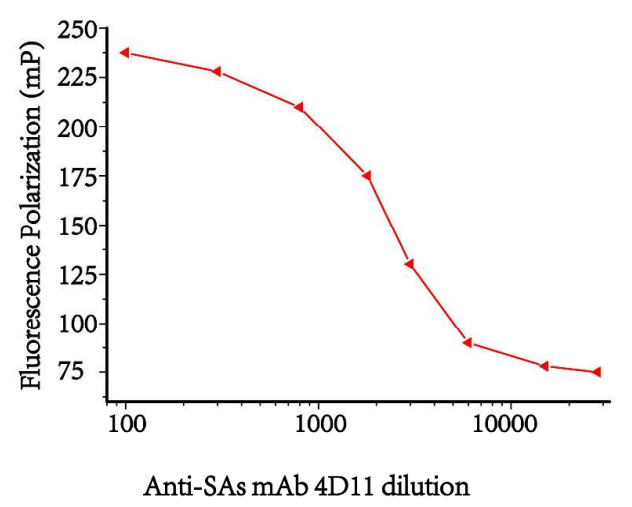

B

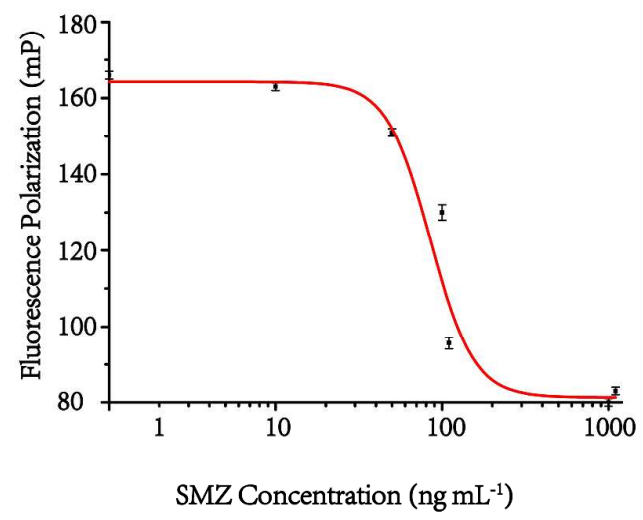

Figure S7. (A) Dilution curve for anti-SAs mAb4D11 with tracer BS-AMF, and (B) FPIA calibration curves for SMZ using tracer BS-AMF.

The CR of the FPIA for sulfonamides is shown in Table S1.

Table S1. FPIA cross-reactivity (CR) results for sulfonamides

\begin{tabular}{lcc}
\hline Sulfonamides (SAs) & $\mathrm{IC}_{50}\left(\mathrm{ng} \mathrm{mL}^{-1}\right)$ & $\mathrm{CR}(\%)$ \\
\hline Sulfamethazine (SMZ) & 98.7 & $100 \%$ \\
Sulfadimethoxine (SDM) & 11.36 & 868.67 \\
Sulfaquinoxaline (SQX) & 259.76 & 37.87 \\
Sulfamethoxazole (SMX) & 68.58 & 143.93 \\
\hline
\end{tabular}

The mean recoveries of three spiked concentrations at 100,150 , and $200 \mu \mathrm{g} \mathrm{L}^{-1}$

were $125.3,91.2$ and $75.4 \%$ with CV values below $16.3 \%$, respectively. 


\section{References}

(1) Peng, Z. A.; Peng, X. Formation of High-Quality CdTe, CdSe, and CdS Nanocrystals Using CdO as Precursor. J. Am. Chem. Soc. 2001, 123, 183-184.

(2) Xie, H.-Y.; Liang, J.-G.; Liu, Y.; Zhang, Z.-L.; Pang, D.-W.; He, Z.-K.; Lu, Z.-X.; Huang, W.-H. Preparation and Characterization of Overcoated II-VI Quantum Dots. J. Nanosci. Nanotechnol. 2005, 5, 880-886.

(3) Li, J. J.; Wang, Y. A.; Guo, W.; Keay, J. C.; Mishima, T. D.; Johnson, M. B.; Peng, X. Large-Scale Synthesis of Nearly Monodisperse CdSe/CdS Core/Shell Nanocrystals Using Air-Stable Reagents via Successive Ion Layer Adsorption and Reaction. J. Am. Chem. Soc. 2003, 125, 12567-12575.

(4) Xie, R.; Kolb, U.; Li, J.; Basché, T.; Mews, A. Synthesis and Characterization of Highly Luminescent CdSe-Core CdS/ $\mathrm{Zn}_{0.5} \mathrm{Cd}_{0.5} \mathrm{~S} / \mathrm{ZnS}$ Multishell Nanocrystals. J. Am. Chem. Soc. 2005, 127, 7480-7488.

(5) Speranskaya, E. S.; Beloglazova, N. V.; Lenain, P.; De Saeger, S.; Wang, Z.; Zhang, S.; Hens, Z.; Knopp, D.; Niessner, R.; Potapkin, D. V.; Goryacheva, I. Y. Polymer-Coated Fluorescent CdSe-Based Quantum Dots for Application in Immunoassay. Biosens. Bioelectron. 2014, 53, $225-231$.

(6) Reiss, P.; Protière, M.; Li, L. Core/Shell Semiconductor Nanocrystals. Small 2009, 5, 154168.

(7) Tao, X.; Jiang, H.; Yu, X.; Zhu, J.; Wang, X.; Wang, Z.; Niu, L.; Wu, X.; Shen, J. An Ultrasensitive Chemiluminescence Immunoassay of Chloramphenicol Based on Gold Nanoparticles and Magnetic Beads. Drug Test. Anal. 2013, 5, 346-352.

(8) Liang, X.; Ni, H.; Beier, R. C.; Dong, Y.; Li, J.; Luo, X.; Zhang, S.; Shen, J.; Wang, Z. Highly Broad-Specific and Sensitive Enzyme-Linked Immunosorbent Assay for Screening Sulfonamides: Assay Optimization and Application to Milk Samples. Food Anal. Methods 2014, 7, 1992-2002.

(9) Zhang, H.; Mi, T.; Khan, O. Y.; Sheng, Y.; Eremin, S. A.; Beier, R. C.; Zhang, S.; Shen, J.; Wang, Z. Fluorescence Polarization Immunoassay Using IgY Antibodies for Detection of Valnemulin in Swine Tissue. Anal. Bioanal. Chem. 2015, 407, 7843-7848. 
(10) Wang, Z.-H.; Mi, T.-J.; Shen, J.-Z. Advance in Fluorescence Polarization Immunoassay for the Determination of Mycotoxins in Cereals and Related Products. Sci. Agric. Sin. 2012, 45, $4862-4872$.

(11) Mi, T., Liang, X.; Ding, L.; Zhang, S.; Eremin, S. A.; Beier, R. C.; Shen, J.; Wang, Z. Development and Optimization of a Fluorescence Polarization Immunoassay for Orbifloxacin in Milk. Anal. Methods 2014, 6, 3849-3857. 